\title{
HPV and Cervical Cancer Prevention Counseling With Younger Adolescents: Implications for Primary Care
}

\author{
Andrew L. Sussman, PbD, MCRP \\ Deborab Helitzer, ScD ${ }^{1}$ \\ Margaret Sanders, $P b D^{1}$ \\ Brisa Urquieta, BUS ${ }^{1}$ \\ Melina Salvador, $M A^{2}$ \\ Khadidiatou Ndiaye, $M A^{3}$ \\ 'Department of Family and Community \\ Medicine, University of New Mexico, \\ Albuquerque, NM \\ ${ }^{2}$ Department of Psychiatry, University \\ of New Mexico, Albuquerque, NM \\ ${ }^{3}$ Department of Communication Arts and \\ Sciences, The Pennsylvania State Univer- \\ sity, University Park, Pa
}

AC Annals Journal Club selection; see inside back cover or http://www. annfammed.org/AJC/.

Conflicts of interest: none reported

\section{CORRESPONDING AUTHOR}

Deborah Helitzer, ScD

Department of Family and Community Medicine MSC09 5040

1 University of New Mexico

Albuquerque, NM 87131

helitzer@salud.unm.edu

\begin{abstract}
PURPOSE Primary care clinicians will continue to play an important role in cervical cancer prevention, particularly with regard to administration of the newly licensed human papillomavirus (HPV) vaccine and continued administration of Papanicolaou tests. Little is known about the factors that influence cervical cancer prevention counseling, particularly in the adolescent encounter. We conducted a qualitative study to better understand the implications for counseling about cervical cancer prevention by primary care clinicians who care for adolescents.
\end{abstract}

METHODS We conducted in-depth interviews with 37 primary care clinicians in New Mexico to understand the context in which they provide anticipatory guidance about sexual health risks as well as their attitudes about counseling for the forthcoming HPV vaccine.

RESULTS Clinicians identified 4 categories of factors related to their counseling experiences with adolescents about HPV: (1) the need to build rapport with adolescent patients, (2) the presumption that adolescent patients engage in high-risk behaviors, (3) the situational delivery and complexity of HPV counseling, and (4) perceptions of clinician and community receptivity to the HPV vaccine.

CONCLUSION Our findings show that conditions of the preadolescent and young adolescent visit pose a challenge to the successful integration of counseling about cervical cancer prevention in primary care. Counseling strategies that are designed to emphasize a preventive focus while including parents in the discussion at the time of vaccination and that are appropriate to populations with different cultural values and beliefs will help to enhance communication about cervical cancer prevention and the particular role of the HPV vaccine.

Ann Fam Med 2007;5:298-304. DOI: 10.1370/afm.723.

\section{INTRODUCTION}

$\mathrm{H}$ uman papillomavirus (HPV) ranks as the most commonly acquired sexually transmitted infection in the United States, and infection with HPV is linked to cervical cancer and genital warts. ${ }^{1,2}$ In June 2006, the first HPV vaccine (Merck) was approved for females aged 9 to 26 years. ${ }^{3}$ Shortly after its approval, the National Advisory Committee on Immunization Practices recommended that the vaccine be given to girls aged 11 to 12 years. ${ }^{4}$

The approval of the HPV vaccine marks the beginning of a new stage in preventing sexually transmitted diseases (STDs) and their sequelae, in this case cervical cancer. Currently, the hepatitis B vaccine, administered during infancy, is the only existing vaccine that prevents a sexually transmitted disease, although several other STD vaccines are in various stages of development. ${ }^{3,5-6}$ Collectively, these vaccines have the potential to serve as powerful disease prevention weapons against chronic illnesses, including cancer. The availability of these vaccines will have important implica- 
tions for adolescents who, because they are at higher risk for contracting these diseases, will likely be the targeted recipients for these preventive strategies. ${ }^{7}$ As reflected in less-than-desirable vaccination rates, however, the availability of an effective vaccine does not ensure its use and acceptability. ${ }^{8-9}$ Previous research, for example, has found that although parents and clinicians generally favor STD and HPV vaccination, both groups have also indicated potential barriers to vaccine dissemination, including a shared reluctance to vaccinate young girls (ie, preadolescents). ${ }^{10-16}$

Because clinicians' recommendations influence parental acceptance of preventive care, it is clear that primary care clinicians will continue to play an important role in the success of HPV and cervical cancer prevention programs. ${ }^{13,16}$ Given the multiple competing demands of the brief clinical encounter, however, clinicians will likely face counseling challenges in their efforts to integrate these vaccines into practice. Much of the research used to assess determinants of clinician attitudes and behavior toward vaccine acceptance has relied on quantitative surveys and the evaluation of structured scenarios to isolate the role of specific factors. ${ }^{16-19}$ Introduction of the HPV vaccine will likely require different counseling approaches and strategies with adolescents and their parents to ensure informed decision making. Before materials are developed to enhance this communication, it is necessary to better understand the broader context of factors that influence counseling about HPV and cervical cancer prevention in the adolescent patient encounter. In the period preceding the approval of the HPV vaccine, we took a first step toward that goal by conducting a qualitative study of primary care clinicians in New Mexico.

\section{METHODS}

\section{Study Design and Setting}

To increase our understanding of factors that may affect delivery of HPV and cervical cancer prevention counseling in primary care, data collection was aimed at examining the current context of counseling with adolescent patients. This qualitative study consisted of a series of semistructured in-depth interviews with primary care clinicians who see adolescent patients in New Mexico. Data collection began in July 2004 and was completed by May 2005.

An advisory board, comprised of 15 content experts in STDs, HPV microbiology, and primary care, guided the research and provided input on topics to be covered, sampling criteria, and development of the interview guide. The protocol for this study was approved by the University of New Mexico Human Research and Review Committee.

\section{Clinician Interviews and Data Analysis} Interview Guide Content

We developed a semistructured clinician interview guide (available as an online-only Supplemental Appendix at http://www.annfammed.org/cgi/content/ full/5/4/298/DC1) to elicit reflection and discussion regarding the full range of issues relevant to HPV and cervical cancer prevention counseling with adolescent patients. Two important considerations influenced the design of the guide: (1) the need to create a discussion that covered the entire context of a typical adolescent patient visit, and (2) the likelihood that HPV and cervical cancer prevention counseling would be part of a broader discussion of anticipatory guidance for STDs. Clinicians were therefore led through a discussion of (1) the general nature of their practice specific to the care of adolescent patients, (2) counseling related to sexual risk taking and STDs, (3) the relationship between cultural and community contexts and sexual risk, (4) counseling related to HPV infection, and (5) their knowledge, attitudes, and perceived barriers to using the HPV vaccine for their patients. Six pilot interviews with clinicians who see adolescent patients helped to refine the final version of the guide.

\section{Sample and Data Collection}

We obtained a list of licensed primary care clinicians from the New Mexico Board of Examiners, and we purposively recruited based on characteristics believed to influence vaccine acceptability, including the clinician's geographic location (rural, frontier, urban), gender, and specialty (nurse-practitioner, school-based health clinician, pediatrician, family physician, internist). Clinicians were sent a letter requesting an interview, after which they were telephoned to confirm interest in and schedule a convenient time and location for the interview. Most interviews lasted between 45 to 60 minutes; all were audio recorded and later transcribed. Respondents were given a $\$ 50$ honorarium for their participation.

\section{Analytic Process}

Following an iterative analytic process, the research team reviewed sets of 3 to 4 transcripts independently, identifying key themes. ${ }^{20}$ The team then met to review these emergent themes and develop a preliminary analytic framework. Subsequent sets of interviews were similarly assessed, with the goal of disconfirming anomalous findings and further clarifying core themes. This process also involved examining the role of factors believed to contribute to HPV and cervical cancer prevention counseling - geography, gender, and specialty — and modifying the interview guide and sampling strategy accordingly. Ongoing data collection and analysis continued until 37 interviews had been conducted, at which point 
the research team reached consensus that the full range of organizing themes had been identified. The qualitative data analysis software program NVivo2 was used to facilitate coding and text retrieval. ${ }^{21}$

\section{RESULTS}

A total of 37 clinicians agreed to be interviewed. Demographic information for participants is included in Table 1.

\section{Context of the Adolescent Visit}

In the first part of the qualitative interviews, clinicians were asked to describe a typical well-child visit with their adolescent patients (aged 10 to 20 years), with particular attention to anticipatory guidance for risk behaviors, such as substance use, sexual activity, and violence. Clinicians identified the challenge of addressing a wide range of topics during the brief clinical encounter.

I usually start by asking the parents questions on developmental milestones, how they're doing in school, are they in any sports, what their activities and interests are. From there we go to the physical exam. I'm real careful to explain what we're doing as we do it. And then after that we go into anticipatory guidance, where we talk about ... with teenagers especially, I want to make sure I touch on drugs, alcohol, tobacco, and sexual activity [female nurse-practitioner].

Although many clinicians agreed with the importance of these anticipatory guidance goals, they also expressed a need to tailor the specific content of a given encounter based on a process of reading cues from the adolescent. Clinicians characterized the ultimate content of the encounter as a negotiation based on their presumption of the adolescent's risk level and the degree of rapport existing between the clinician, patient and, if present, the parent. They reported that

\begin{tabular}{|lc|}
\hline $\begin{array}{l}\text { Table 1. Demographic Characteristics of Primary } \\
\text { Care Clinicians }(\mathbf{n}=\mathbf{3 7})\end{array}$ \\
\hline Characteristics & No. (\%) \\
\hline Gender & \\
Female & $32(86)$ \\
Male & $5(14)$ \\
Specialty & \\
Family practice & $13(35)$ \\
Obstetrics-gynecology & $12(32)$ \\
Pediatrics & $5(14)$ \\
Other & $7(19)$ \\
New Mexico Department of & \\
Health Public health districts & $14(38)$ \\
District 1 (Albuquerque) & $11(30)$ \\
District 2 (Santa Fe) & $6(16)$ \\
District 3 (Las Cruces) & $6(16)$ \\
District 4 (Roswell) & \\
\hline
\end{tabular}

the topics they discussed with younger adolescents (aged 10 to 12 years) were likely to be quite different from those they discussed with patients aged 13 to 15 years and especially different from those they discussed with patients aged 16 to 19 years. For example, sexual risk taking was not likely to be discussed if the child did not give cues that she or he was ready for this discussion, especially at younger ages.

The importance of building rapport and trust with their adolescent patients was a prominent theme. During encounters in which the parent was present, clinicians created the opportunity to speak privately with the adolescent as a way to enhance communication about potentially sensitive topics.

But if I find that this person is not opening up, I'll send the parents out. And I tell the girls, you know, [that in] the state of New Mexico ... they can come to me for STD counseling and treatment, for contraception, for pregnancy ... without parental consent. That whatever they tell me is confidential, and that I do not have to tell their parents. And then it's sort of like, "whew," you know, and then they'll start talking [female pediatrician].

A lot of times you can tell by the demeanor of the child, how they're answering you, if they really want the parents to not be in there ... if they've got something that they want to talk to you about, or whatever. And so a lot of times, like during school sports physicals, I'll ask the parents to step out. Sometimes they refuse, but if they don't, then I can at least talk to the child a little bit [female nurse-practitioner].

\section{Presumption of Adolescent Risk}

A striking theme that emerged from the clinician interviews was the presumption that adolescents engage in risky health behaviors-sexual, safety, substance abuse - at high rates.

That 10 - to 20 -year-old age-group, I mean, their risky sexual behavior is unbelievable. I mean, multiple partners, multiple, multiple partners, alcohol use, meth ... and the more risk factors that you put into it ... [female nurse-practitioner]

Given the presumption that many adolescents begin to engage in risky sexual behaviors by the age of 13 or 14 years, clinicians identified a need to prioritize this counseling. We found a range of approaches used by clinicians to gauge the readiness of the adolescent patient before engaging in a discussion about sexual behavior. For example, some clinicians determined the need for counseling as a result of the adolescents' responses to questions about his or her social life.

... and from their goals, then I start talking to them about risk factors, if they drink alcohol or use any drugs, if they are sexually active, if they've thought about it, if they have a boyfriend or girlfriend. I don't assume that they are going to tell me the truth [female physician's assistant]. 
Other clinicians expressed a more aggressive approach:

... I prioritize intercourse - sexually transmitted diseasesbecause it is very prevalent in the population. I can say that I probably start most of my females on the pill that walk in the door without being on it, because they have been sexually active and their parents don't know or they haven't felt comfortable coming in to speak to anyone about it [female family physician].

\section{HPV Counseling}

Clinicians reported that they seldom initiated counseling about HPV infection, either separately or as part of the broader menu of STD counseling, because of (1) the complexity of HPV infection counseling and the low level of baseline knowledge held by most adolescents about HPV infection, (2) their discomfort that resulted from a limited knowledge base of HPV, and (3) time constraints in the typical adolescent visit.

I say genital warts when I counsel them about STDs. I don't normally say HPV because I don't think they quite understand. When I say prevention of STDs, including gonorrhea, Chlamydia, genital warts, and AIDS, abstinence and condoms - that is what is going to protect you. But I don't extend to cervical dysplasia, you know, cervical cancer, because I think that is too confusing for them, but they know what genital warts are [female family physician]

Instead, HPV infection was more likely to be addressed in relation to a teachable moment, such as the discovery of genital warts or when reporting findings from an abnormal Papanicolaou (Pap) smear.

A lot of conversation is generated if they have a bump they think is something, then we go into, well, what is a genital wart? Why is it dangerous? And I tell them that usually if we can see a wart visibly, there may be more than one type of HPV present.... And we go into it more in depth also if they have an abnormal Pap-we talk to them a lot about HPV and what needs to be done, how we're going to have to follow them, and things like that [female nurse-practitioner].

Despite probing specifically about counseling of male patients about HPV infection, the interview data do not provide evidence of examples of how clinicians provide anticipatory guidance to male patients about the link between cervical cancer and HPV infection. Clinicians indicated the lack of counseling opportunities to engage their male patients about this issue as the primary reason for this gap.

The boys are more difficult, because there's nothing leading into that, you know. Many times if you say, "Is there any other problem that we need to address right now?" it just goes above their head. Or they want it to go above their head. And so it's more difficult [female nurse-practitioner].
Clinician Perceptions of HPV Vaccine Receptivity Clinician reactions to the HPV vaccine and their perceptions of its receptivity in the communities they serve were generally mixed. As a scientific advance in preventing cervical cancer, clinician receptivity for the vaccine was high. Furthermore, clinicians spoke to benefits of the vaccine in reducing the emotional costs for women who experience cervical abnormalities.

I mean that's huge, we've done a great job as far as screening with Paps and getting therapy, but if we can prevent that, that would be great. Not only as far as costs, but to tell somebody and have to explain to them they have low-grade dysplasia and we need to do treatment, I mean, its overwhelming, and they don't, they are like, "What does it mean, am I going to die?" I think it would be very beneficial [female obstetrician-gynecologist].

Some clinicians, however, were concerned that the HPV vaccine would give adolescents a false sense of protection.

I guess the one downfall is when somebody says, "Well, I'm vaccinated against this [HPV], I'm not worried about it." Are people gonna be less likely to be concerned about other diseases, gonorrhea, Chlamydia, and those things [male family physician]?

Additionally, clinicians expressed some level of concern regarding the vaccine's receptivity in the communities they serve based on cultural beliefs regarding sexual activity.

That would be difficult because you would have to get parental consent (to give the HPV vaccine). I think that would be very difficult. Because a lot of my patients would deny that they are thinking of becoming sexually active, and a lot of the parents don't know, even if there has been some open door there [female family physician].

When asked about what age to vaccinate for HPV, I don't have an answer. I mean, I think if you said 16 across the board, I think you would be severely limiting the spread, but you may miss a small percentage. But I don't know that any younger is gonna be culturally accepted. Some parents are not going to feel comfortable about talking to the kids, some are not gonna be comfortable until they're married at 28 or 30 [male obstetrician-gynecologist].

Factors that we initially believed would influence vaccine acceptability, such as gender of clinician, geography, and type of practice, did not emerge as important or discriminating in our sample. Female clinicians, for example, expressed greater enthusiasm about the vaccine and its potential for cervical cancer prevention but were equally anxious about how to counsel about HPV infection. Some rural clinicians discussed the conservative nature of their communities and the potential push-back by parents when offered the vaccine, but this issue was also raised by clinicians 
in urban settings. Finally, while pediatricians more consistently raised the issue about offering the vaccine to younger patients as a potential barrier, we found that clinicians across specialties shared similar concerns.

\section{DISCUSSION}

This study provides a step toward a better understanding of the factors that influence HPV and cervical cancer prevention counseling decisions of primary care clinicians with their adolescent patients. Findings from this qualitative study show that clinicians believe active and aggressive sexual risk-taking counseling is necessary for their adolescent patients because of perceived high levels of risky behaviors. Clinicians expressed a situational approach to STD counseling, reading patient and parental cues to identify appropriate counseling topics and tailoring the message accordingly. Notably, clinicians reported that HPV infection counseling was typically not included as part of general anticipatory guidance about STD prevention. When clinicians did counsel for HPV infection, they reported initiating this discussion in association with teachable moments, such as when reporting abnormal Papanicolaou smear results or finding genital warts. Such teachable moments occurred, however, when the adolescent was much older than the age recommended for HPV vaccination. Although our study took place before the approval and release of the HPV vaccine, we believe our findings have important implications for assessing the degree to which counseling about HPV and cervical cancer prevention fit in primary care.

\section{Application}

Primary care is positioned at a key intersection in the immunization delivery process, and primary care clinicians will play a central role in parents' knowledge and attitudes about HPV infection and their receptivity toward the HPV vaccine. ${ }^{22-25}$ Although findings from our study support the need for disseminating information about the HPV vaccine to primary care clinicians, other counseling factors related to the context of the adolescent encounter may be equally, if not more, important when considering the vaccine's acceptance among patients and their families. ${ }^{26,27}$ We have identified 4 potential challenges related to the nature of prospective discussions about HPV infection with younger and older adolescents and their parents.

The first challenge relates to the current context in which clinicians report counseling about HPV infection and its implications for discussing the vaccine. Clinicians in our study indicated that they did not often include HPV or cervical cancer prevention in their adolescent anticipatory guidance counseling about STDs; rather, they addressed it as a result of a more acute problem, such as when the patient had abnormal Papanicolaou smear findings. Furthermore, the perception that HPV infection is a particularly complicated topic to discuss generally leads clinicians to give priority to other important topics during the adolescent visit. The challenge of discussing HPV infection is supported by research indicating a lack of understanding about this infection by adults and adolescents. ${ }^{28,29}$ The introduction of the HPV vaccine, however, will likely increase the need for counseling about HPV and cervical cancer prevention at much younger age, before the adolescent is sexually active, and thus change the context in which the discussion of prevention is introduced. Additionally, vaccine recipients will need to be reminded that the current vaccine is not effective against all HPV types implicated in cervical cancer. A related communication issue involves the strategic decision of how to counsel most effectively about the vaccine as a tool for cervical cancer prevention or STD prevention. Counseling strategies that incorporate various dimensions of communication will therefore likely be helpful to primary care clinicians.

The second challenge for primary care clinicians involves the recommended age for giving the HPV vaccine and communication with parents. Although females aged 9 to 26 years are eligible to receive the HPV vaccine, it will achieve the greatest benefit if administered before initiation of sexual activity; therefore, the vaccine is recommended to be given to girls aged 11 to 12 years. ${ }^{4,6,13,30,31}$ Earlier research about the attitudes of clinicians and parents toward STD vaccines, however, has consistently shown a preference for vaccinating either infants or older adolescents. ${ }^{12,17,18}$ Further complicating the issue of when to administer the vaccine are our study findings that (1) parents accompany younger adolescents to most of their primary care visits, and (2) clinicians indicated a preference to have the parents leave the room to enhance communication with the adolescent about potentially sensitive topics. Counseling for the HPV vaccine will often be conducted with younger adolescents while in the presence of a parent or caregivers, and these adolescents are currently not regularly exposed to anticipatory guidance about STDs. Communication about the HPV vaccine may therefore necessitate a shift in counseling patterns and dynamics to address sexual activity with preadolescent girls while including parents in these discussions.

Third, clinicians identified the need to consider factors external to the patient-clinician encounter, such as the diverse cultural and religious beliefs across communities, the impact of mass media advertising 
campaigns, and competing patient demands. Although other research has found high levels of perceived acceptability of HPV vaccination among parents, ${ }^{2,5,11,14}$ we found that clinicians expressed stronger concerns about receptivity to the vaccine in the mostly Hispanic and Native American populations they served. These populations have not been well represented in previous research examining knowledge, attitudes, and beliefs about the vaccine. Now that the vaccine has been released, it will be important to assess the responses from various social, religious, and political factions, as it is unclear how anticipatory cervical cancer prevention counseling will affect disparate cultural groups. Presenting information in ways that are sensitive to a range of cultural norms and social values associated with sexual activity in adolescents will likely play a role in enhancing vaccine acceptance.

Finally, compliance with the 3 -dose HPV vaccine series is a potential barrier, given that many adolescents may receive the first dose during an acute or previously scheduled visit but then face challenges in returning for subsequent doses. ${ }^{32}$ Inadequate reimbursement from insurance carriers also appears to be placing a strain on limited clinic resources. Accordingly, financial and logistical challenges for busy primary care practices to feasibly provide the vaccine and ensure that patients return for 2 additional visits within a 6 -month period will need to be addressed.

\section{Limitations}

This research was conducted among New Mexico primary care clinicians, including those serving the state's diverse ethnic and geographically distributed populations. It is possible that their views toward HPV infection counseling differ from those of clinicians in other parts of the country. We believe, however, that the issues identified by these clinicians are similar across practice settings and geographic location. Female clinicians were more likely to participate in our study, so our results may represent a more femalebiased perspective. Additionally, in the interviews we defined "adolescent patients" as patients aged 10 to 19 years. The vaccine has been approved for 9-year-old girls; therefore, our findings do not directly apply to counseling perspectives with these patients. Given the concerns we report regarding communication about sensitive matters with parents of younger adolescents, however, we believe that our findings are equally (if not more) relevant for these patients. It is also important to note that the timing of the study (1 to 2 years before vaccine approval) is relevant to our findings. It is likely that the recent dissemination of clinical information associated with the vaccine's release will lead to evolving perspectives of the results presented herein.
The primary purpose of this study, however, was to identify contextual factors that influence HPV and cervical cancer prevention counseling in the adolescent primary care encounter. We believe that these factors are relatively constant and our findings have direct relevance to the development of effective strategies aimed at enhancing communication among clinicians, adolescents, and their parents about the HPV vaccine.

In this qualitative study, we have taken a first step toward identifying primary care clinicians' perspectives about the implications of cervical cancer prevention and HPV vaccine counseling for primary care. These findings suggest that several factors will play an important role in efforts to integrate the HPV vaccine successfully in primary care. Most prominently, it will be necessary to develop counseling strategies that emphasize a preventive focus, to include parents in the discussion at the time of vaccination, and to take into account the needs of populations with different cultural values and beliefs. Further research to craft these approaches will be necessary to support informed decision making among patients and their families and enhance the benefit of this exciting advance.

To read or post commentaries in response to this article, see it online at http://www.annfammed.org/cgi/content/full/5/4/298.

Key words: Human papillomavirus; papillomavirus vaccines; cancer vaccines; anticipatory guidance; patient acceptance of health care; qualitative research, evaluation studies; primary health care; sexually transmitted diseases; adolescent health services

Submitted August 3, 2006; submitted, revised, May 10, 2007; accepted May 20, 2007.

Funding support: This study was supported by the University of New Mexico Cancer Research and Treatment Center.

Acknowledgments: We acknowledge and appreciate the primary care clinicians who gave their time to participate in this study. Members of the Advisory Board provided important oversight throughout the study. We would also like to acknowledge Cosette Wheeler, PhD, for her expert consultation, and Lavinia Nicolae, MA, for her role in interviewing clinicians for this study.

\section{References}

1. Weinstock H, Berman S, Cates W, Jr. Sexually transmitted diseases among American youth: incidence and prevalence estimates, 2000. Perspect Sex Reprod Health. 2004;36(1):6-10.

2. Olshen E, Woods ER, Austin SB, Luskin M, Bauchner H. Parental acceptance of the human papillomavirus vaccine. J Adolesc Health. 2005;37(3):248-251.

3. Koutsky LA, Ault KA, Wheeler CM, et al. A controlled trial of a human papillomavirus type 16 vaccine. $N$ Engl J Med. 2002;347(21):1645-1651.

4. Centers for Disease Control. ACIP Provisional Recommendations for the Use of Quadrivalent HPV Vaccine. http://www.cdc.gov/nip/recs/ provisional_recs/hpv.pdf. Accessed: December 21, 2006. 
5. Mays RM, Sturm LA, Zimet GD. Parental perspectives on vaccinating children against sexually transmitted infections. Soc Sci Med. 2004;58(7):1405-1413.

6. Stanberry LR, Spruance SL, Cunningham AL, et al. Glycoprotein-D-adjuvant vaccine to prevent genital herpes. N Engl J Med. 2002;347(21):1652-1661.

7. Trends in sexual risk behaviors among high school students--United States, 1991-2001. MMWR Morb Mortal Wkly Rep. 2002;51(38): 856-859.

8. Gust DA, Strine TW, Maurice E, et al. Underimmunization among children: effects of vaccine safety concerns on immunization status. Pediatrics. 2004;114(1):e16-22.

9. Smith PJ, Chu SY, Barker LE. Children who have received no vaccines: who are they and where do they live? Pediatrics. 2004;114(1):187-195.

10. Kahn JA, Rosenthal SL, Hamann T, Bernstein DI. Attitudes about human papillomavirus vaccine in young women. Int J STD AIDS. 2003;14(5):300-306

11. Davis K, Dickman ED, Ferris D, Dias JK. Human papillomavirus vaccine acceptability among parents of 10- to 15-year-old adolescents. J Low Genit Tract Dis. 2004;8(3):188-194.

12. Waller J, Marlow LA, Wardle J. Mothers' attitudes towards preventing cervical cancer through human papillomavirus vaccination: a qualitative study. Cancer Epidemiol Biomarkers Prev. 2006;15(7):1257-1261.

13. Zimet GD. Improving adolescent health: focus on HPV vaccine acceptance. J Adolesc Health. 2005;37(6 Suppl):S17-23.

14. Dempsey AF, Zimet GD, Davis RL, Koutsky L. Factors that are associated with parental acceptance of human papillomavirus vaccines: a randomized intervention study of written information about HPV Pediatrics. 2006;117(5):1486-1493.

15. Davis K, Dickman ED, Ferris D, Dias JK. Human papillomavirus vaccine acceptability among parents of 10- to 15-year-old adolescents. J Low Genit Tract Dis. 2004;8(3):188-194.

16. Mays RM, Zimet GD. Recommending STI vaccination to parents of adolescents: the attitudes of nurse practitioners. Sex Transm Dis. 2004;31(7):428-432

17. Raley JC, Followwill KA, Zimet GD, Ault KA. Gynecologists' attitudes regarding human papilloma virus vaccination: a survey of Fellows of the American College of Obstetricians and Gynecologists. Infect Dis Obstet Gynecol. 2004;12(3-4):127-133.

18. Riedesel JM, Rosenthal SL, Zimet GD, et al. Attitudes about human papillomavirus vaccine among family physicians. J Pediatr Adolesc Gynecol. 2005;18(6):391-398
19. Kahn JA, Zimet GD, Bernstein DI, et al. Pediatricians' intention to administer human papillomavirus vaccine: the role of practice characteristics, knowledge, and attitudes. J Adolesc Health. 2005;37(6):502-510.

20. Crabtree B, Miller W. Doing Qualitative Research. 2nd ed. Thousand Oaks, Calif: Sage Publications, Inc; 1999.

21. NVivo [computer program]. Version 2. Doncaster, Victoria, Australia: QSR International; 2002

22. Freed GL, Clark SJ, Hibbs BF, Santoli JM. Parental vaccine safety concerns. The experiences of pediatricians and family physicians. Am J Prev Med. 2004;26(1):11-14.

23. Gellin BG, Maibach EW, Marcuse EK. Do parents understand immunizations? A national telephone survey. Pediatrics. 2000;106(5):10971102.

24. Gust DA, Kennedy A, Shui I, Smith PJ, Nowak G, Pickering LK. Parent attitudes toward immunizations and healthcare providers the role of information. Am J Prev Med. 2005;29(2):105-112.

25. DiMatteo MR. Future directions in research on consumer-provider communication and adherence to cancer prevention and treatment. Patient Educ Couns. 2003;50(1):23-26.

26. McMurray R, Cheater FM, Weighall A, Nelson C, Schweiger M, Mukherjee $S$. Managing controversy through consultation: a qualitative study of communication and trust around MMR vaccination decisions. Br J Gen Pract. 2004;54(504):520-525.

27. Rosenthal SL, Stanberry LR. Parental acceptability of vaccines for sexually transmitted infections. Arch Pediatr Adolesc Med. 2005:159(2):190-192.

28. Holcomb B, Bailey JM, Crawford K, Ruffin MT. Adults' knowledge and behaviors related to human papillomavirus infection. J Am Board Fam Pract. 2004;17(1):26-31.

29. Mays RM, Zimet GD, Winston Y, Kee R, Dickes J, Su L. Human papillomavirus, genital warts, Pap smears, and cervical cancer: knowledge and beliefs of adolescent and adult women. Health Care Women Int. 2000;21(5):361-374

30. Goldie SJ, Kohli M, Grima D, et al. Projected clinical benefits and cost-effectiveness of a human papillomavirus 16/18 vaccine. J Nat Cancer Inst. 2004;96(8):604-615.

31. Rosenthal SL. Protecting their adolescents from harm: parental views on STI vaccination. J Adolesc Health. 2005;37(3):177-178.

32. Middleman AB, Rosenthal SL, Rickert VI, Neinstein L, Fishbein DB, D'Angelo L. Adolescent immunizations: a position paper of the Society for Adolescent Medicine. J Adolesc Health. 2006;38(3):321-327. 